\title{
Defective activities, but not secretions, resulting from gene point mutations of human mannan-binding lectin
}

\author{
YING LIU, FENG-LING LIU, ZHI-JUN BAI, NA ZHAO, LI-YUN ZHANG, XIAO LU and ZHENG-LIANG CHEN
}

Department of Immunology, School of Basic Medical Sciences, Southern Medical University, Guangzhou 510515, P.R. China

Received July 20, 2011; Accepted February 3, 2012

DOI: $10.3892 / \mathrm{mmr} .2012 .782$

\begin{abstract}
Human mannan-binding lectin (MBL) plays a pivotal role in innate immunity. Substantial literature supports the belief that three point mutations, CGT52TGT, GGC54GAC and GGA57GAA, in the collagen-like region (CLR) of the human MBL gene, are associated with increased susceptibility to infection, autoimmunity and carcinogenesis. To investigate the mechanisms of MBL deficiency, human wild-type and three variant MBL genes were expressed in COS-7 and Chinese hamster ovary $(\mathrm{CHO})$ cells. Results showed that no apparent differences were found among the levels of gene transcription and protein secretion of four forms of MBL. However, the degree of oligomerization of variant forms of MBL was found to be much lower than that of recombinant human wild-type MBL. The ability of variant MBL proteins to bind mannan was much weaker than that of the wild-type MBL protein, and the MBL variants failed to effectively activate the complement lectin pathway. These data suggested that a lower order oligomer, but not decreased plasma levels of MBL, may be the main result of MBL gene mutations and may be associated with immunodeficiency.
\end{abstract}

\section{Introduction}

Mannan-binding lectin (MBL; also known as mannose-binding lectin), a member of the family of collectins (C-type lectins with a collagen-like domain), is considered a classical pattern recognition molecule of innate immunity. MBL recognizes

Correspondence to: Professor Zheng-Liang Chen, Department of Immunology, School of Basic Medical Sciences, Southern Medical University, Guangzhou 510515, P.R. China

E-mail: zhlchen@fimmu.com

Abbreviations: MBL, mannan-binding lectin; CRD, carbohydrate recognition domain; CLR, collagen-like region; MASP, MBL-associated serine protease; RA, rheumatoid arthritis; SLE, systemic lupus erythematosus; $\mathrm{CHO}$, Chinese hamster ovary; ELISA, enzyme-linked immunosorbent assay; PVDF, polyvinylidene difluoride; HRP, horseradish peroxidase

Key words: mannan-binding lectin, collagen-like region, mutation, oligomerization, secretion, immunodeficiency and binds to conserved carbohydrate motifs, such as mannose, mannan, N-acetyl-mannosamine, $\mathrm{N}$-acetyl-D-glucosamine and fucose, MBL and is present on the surface of a variety of micro-organisms via its carbohydrate recognition domain (CRD) in a calcium-dependent manner; its biological effect is thought to be mediated by a collagen-like region (CLR) $(1,2)$. MBL constitutes a crucial part of innate immunity as it triggers immediate cell lysis or phagocytosis by initiating the lectin pathway of complement activation. MBL also promotes phagocytosis by directly binding to cell surface receptors without the involvement of the complement $(3,4)$. The lectin pathway activation is mediated by three MBL-associated serine proteases (MASPs), MASP1, MASP-2 and MASP-3 (5). MBL is also involved in the clearance of abnormal self-components, including cell debris and apoptotic cells (6).

Mounting evidence suggests that MBL gene mutations, a common genetic disorder, are associated with increased susceptibility to a broad spectrum of infections, particularly when immunity is already compromised by immunological immaturity, comorbidity or chemotherapy $(7,8)$. MBL mutations may also contribute to the morbidity of autoimmune disorders, such as rheumatoid arthritis (RA) and systemic lupus erythematosus (SLE) $(9,10)$. Moreover, variant MBL alleles are associated with disease progression in concomitant diseases, such as cystic fibrosis and chronic granulomatous disease $(11,12)$. The wildtype human MBL2 gene (MBL1 is a pseudogene) is referred to as A, and three variant alleles in CLR are B (GGC54GAC), C (GGA57GAA) and D (CGT52TGT), resulting in the amino acid changes from Gly to Asp (34Asp, mature peptide without a signal sequence of 20 amino acids), Gly to Glu (37Glu) and Arg to Cys (32Cys), respectively. The frequencies of these mutations vary among ethnic groups, but are notably high overall. Allele B is undoubtedly the most common basis for low MBL levels in Europeans, with frequency ranging from 0.11 to 0.15 . $\mathrm{C}$ is found most frequently in sub-Saharan African populations, with frequency ranging from 0.23 to 0.29 , but D is rare in all populations examined thus far (0.06-0.08) and appears to be limited to Caucasian and northern East African populations (13). Our previous study showed that the frequencies of allele B are $0.138,0.168$ and 0.100 in Chinese Han, Uygur and Pai (Bai) populations, respectively, but alleles $\mathrm{C}$ and $\mathrm{D}$ were not detected in these populations $(14,15)$.

It is widely accepted that possession of variant alleles conferring low MBL concentrations is associated with a high risk of recurrent infections. However, there is a large variation 
of MBL levels in individuals with identical genotypes. For example, it was found that the plasma levels range from 0 to $5 \mathrm{ng} / \mathrm{ml}$ (median 1.2) in individuals homozygous for allele A, but from 0 to $1.2 \mathrm{ng} / \mathrm{ml}$ (median 0.2 ) in individuals heterozygous for allele B (16). Therefore, the mechanisms involved in the correlation between MBL mutations and susceptibility to infection have yet to be defined. Whether or not the MBL deficiency results from the decreased plasma level or the impaired functions should also be investigated.

The aim of the present study was to preliminarily define the associations of human MBL variant alleles with immunodeficiency, including the secretion levels and functions of MBL. We expressed the wild-type as well as variant forms of MBL in COS-7 and Chinese hamster ovary (CHO) cells by transient and stable transfection, respectively. Antigenic MBL levels were analyzed by indirect and sandwich enzyme-linked immunosorbent assay (ELISA), the molecular weights of MBL proteins by SDS-PAGE and western blotting, and functional activities by the mannan-binding, MASP-binding and $\mathrm{C} 4$ deposition assays. The results indicated that the mutations in the MBL gene may affect the oligomer formation of MBL, which in turn leads to a defective ability to bind ligand and to activate the complement lectin pathway, rather than synthesis or secretion of the protein.

\section{Materials and methods}

Cloning and vector construction of MBL genes. The intact cDNA encoding MBL polypeptide, including the signal sequence, was amplified from the liver tissue of a 5-month-old Chinese foetus, cloned in a pGEM-T vector. The recombinant plasmid obtained was designated pMBLw. pMBLw was used as a template, and MBL variants GGC54GAC, CGT52TGT and GGA57GAA were created using the Megaprimer-PCR or Takara MutanBEST kit. The products of site-directed mutagenesis were inserted into the pGEM-T vector, and three recombinant plasmids, pMBLm52, pMBLm54 and pMBLm57, were obtained. The target sequences in four recombinant plasmids were amplified by PCR and inserted into eukaryotic expression vectors PcDNA4/HisMaxC. The resulting recombinant expression vectors were designated pcMBLw, pcMBLm52, pcMBLm54 and pcMBLm57, respectively.

Transient expression of wild-type and variant forms of $M B L$ in COS-7 cells. COS-7 cells (ATCC) were plated at a density of $5 \times 10^{4}$ cells $/ \mathrm{ml}$ in 24-well plates (Nunc, Roskilde, Denmark) in Iscove's modified Dulbecco's medium (IMDM; Gibco, Carlsbad, CA, USA) supplemented with $10 \%$ fetal bovine serum (Gibco) for $24 \mathrm{~h}$ prior to transfection. The cells were then transfected with $10 \mu \mathrm{l}$ of Lipofectamine ${ }^{\mathrm{TM}}$ reagent (Invitrogen, Carlsbad,CA, USA) complexed with $1 \mu \mathrm{g}$ of DNA (pcMBLm52, pcMBLm54, pcMBLm57 or pcMBLw). Seventy-two hours later, the culture supernatants were collected and analyzed by sandwich ELISA.

Stable expression of wild-type and variant forms of $M B L$ in $\mathrm{CHO}$ cells. CHO cell transfectants were generated by electroporation of $\mathrm{CHO}$ cells with the recombinant plasmids previously mentioned. Zeocin $(800 \mathrm{mg} / \mathrm{l})$ was added into the cultures for 30 days to select $\mathrm{CHO}$ cells and a further $200 \mathrm{mg} / \mathrm{l}$ were then added for another 30 days to obtain stable
Zeocin-resistant transfectants. The expression of mRNAs was analyzed by RT-PCR. The selected clones were subcloned in 96-well microtitre plates using a limiting dilution method and subsequently incubated in serum-free medium (JRH Bioscience, Victoria, Australia). The recombinant proteins were purified from the culture supernatants by $\mathrm{Ni}^{2+}$-NTA agarose chromatography (Invitrogen) and identified by indirect ELISA.

SDS-PAGE and western blotting. SDS-PAGE was performed using $10 \%$ Tris acetate gels and stained with Coomassie brilliant blue. Western blotting was carried out on polyvinylidene difluoride (PVDF) membrane. Non-specific binding was blocked by incubating the membrane with TBST $[20 \mathrm{mM}$ Tris-HCl, $150 \mathrm{mM} \mathrm{NaCl}, 0.1 \%$ (v/v) Tween-20] containing 5\% (w/v) skim milk at $4^{\circ} \mathrm{C}$ overnight. The membrane was then incubated with anti-MBL monoclonal antibody (mAb) HYB131-11 (Abcam, Cambridge, UK) for $1 \mathrm{~h}$ at room temperature. After washing with TBST, the membrane was further incubated with horseradish peroxidase (HRP)-conjugated goat anti-mouse $\mathrm{IgG}$ for $30 \mathrm{~min}$. The peroxidase reaction was finally performed using TMB as a substrate after washing the membrane.

Indirect ELISA. Flat-bottomed microtitre plates (Nunc) were coated with recombinant wild-type and variant forms of MBL proteins were purified by $\mathrm{Ni}^{2+}$-NTA agarose chromatography at $4^{\circ} \mathrm{C}$ overnight, and then incubated with anti-MBL mAb HYB131-11, anti-MBL-CRD mAb (prepared in our laboratory) or anti-His $\mathrm{mAb}$ (Invitrogen) at $37^{\circ} \mathrm{C}$ for $1 \mathrm{~h}$, followed by HRP-conjugated anti-mouse IgG. The amount of binding proteins was determined by measuring the absorbance at $450 \mathrm{~nm}(\mathrm{~A} 450 \mathrm{~nm})$ using TMB as a substrate for the peroxidase reaction.

Sandwich ELISA. Flat-bottomed microtitre plates were coated with anti-MBL polyclonal antibody (1:100) (prepared in our lab) at $4^{\circ} \mathrm{C}$ overnight, followed by incubation with the culture supernatants of the transient expression system at $37^{\circ} \mathrm{C}$ for $1 \mathrm{~h}$, and HRP-conjugated anti-His polyclonal antibody ( $R \& D$ Systems, Minneapolis, MN, USA) at $37^{\circ} \mathrm{C}$ for $1 \mathrm{~h}$. The amount of binding proteins was determined by measuring A450 nm using TMB as a substrate. The MBL standard was a kind offer from Professor Jens Chr. Jensenius (Aarhus University, Aarhus, Denmark).

Binding of MBL to mannan. Mannan (10 mg/l; Sigma-Aldrich, St. Louis, MO, USA) was placed onto microtitre wells. The wells were then incubated with purified proteins in TBS containing $5 \mathrm{mM} \mathrm{CaCl}_{2}$ and $5 \%(\mathrm{w} / \mathrm{v})$ bovine serum albumin (TBS-Ca-BSA) at $37^{\circ} \mathrm{C}$ for $1 \mathrm{~h}$, anti-MBL mAb HYB131-11 at $37^{\circ} \mathrm{C}$ for $1 \mathrm{~h}$, and HRP-conjugated anti-mouse IgG at $37^{\circ} \mathrm{C}$ for $30 \mathrm{~min}$. The amount of proteins binding to mannan was determined by measuring A450 nm using TMB as a substrate. In certain experiments, proteins were simultaneously incubated with mannan in the presence of mannose, $\mathrm{N}$-acetylglucosamine or EDTA.

Binding of MBL to MASPs. A purified recombinant $\mathrm{N}$ terminal fragment of MASP1 or MASP2 $(10 \mathrm{mg} / \mathrm{l}$, prepared in our laboratory) was placed onto microtitre wells. The wells were then incubated with four MBL proteins and the MBL-CLR 
A

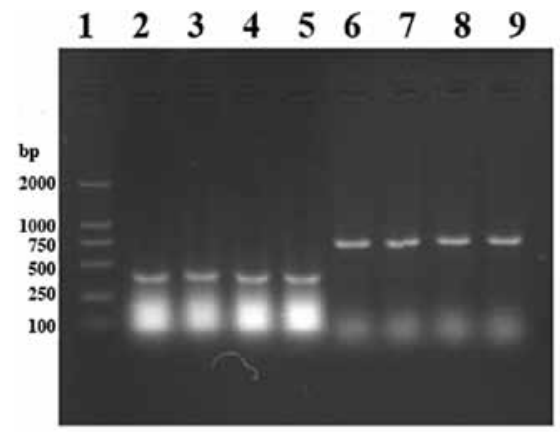

B

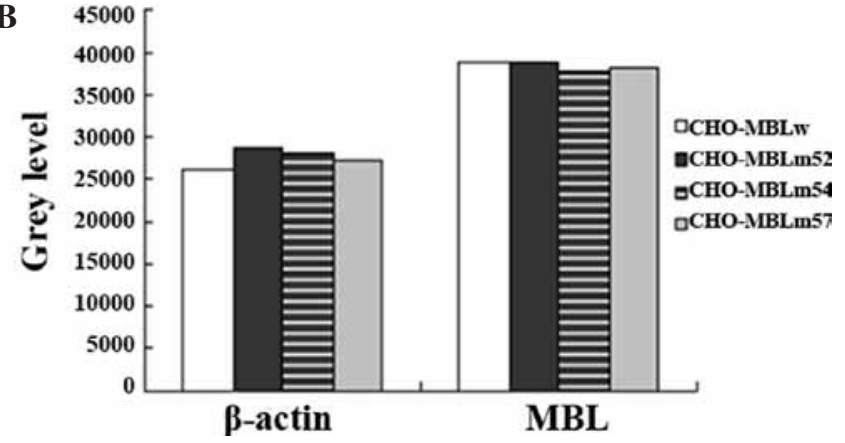

Figure 1. Identification of the transcription levels of four MBL genes. mRNAs were extracted from $1 \times 10^{7}$ transfectant cells and cDNAs were obtained by RT-PCR and analyzed by agarose gel electrophoresis. (A) Electrophoresis of RT-PCR products. Lane 1, DNA marker; lanes 2-5, RT-PCR products of $\beta$-actin; lanes 6-9, RT-PCR products of MBL genes; lanes 2 and 6, CHO-MBLw; lanes 3 and 7, CHO-MBLm52; lanes 4 and 8, CHO-MBLm54 and lanes 5 and 9, CHO-MBLm57. (B) The grey levels of DNA fragments in (A) were determined by the digital gel analysis system.

\section{A $\quad \mathbf{A 5 0} \mathrm{nm}$ value}

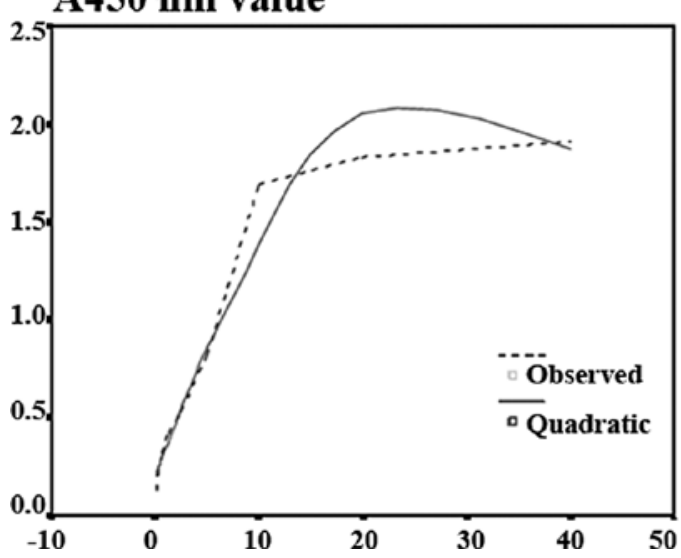

B

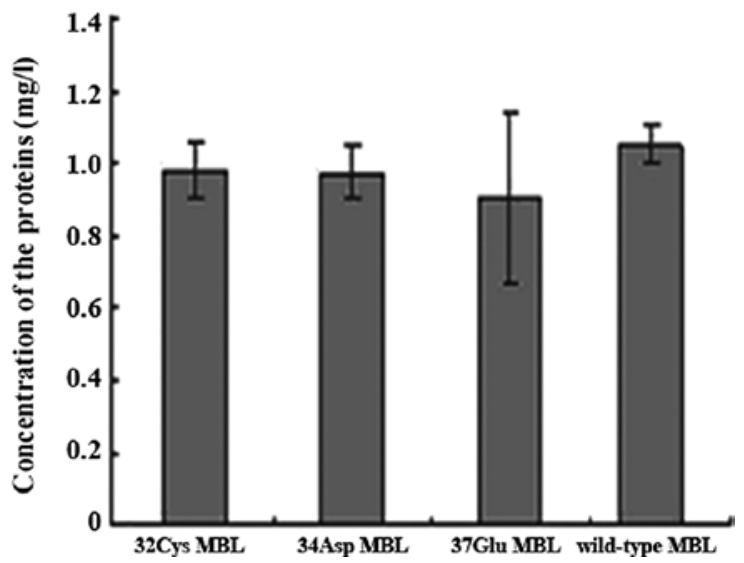

Figure 2. Protein levels in the culture supernatants of the transient expression system. COS-7 cells were plated at a density of $5 \times 10^{4}$ cells $/ \mathrm{ml}$ in $24-w e l l$ plates for $24 \mathrm{~h}$ and then transfected with $1 \mu \mathrm{g}$ of DNA (pcMBLm52, pcMBLm54, pcMBLm57 or pcMBLw). Seventy-two hours later, culture supernatants were collected and analyzed by sandwich ELISA. (A) The MBL standard is diluted from 40 to $0.3125 \mathrm{mg} / 1$. Using the non-linear regression analysis, the protein concentration is independent $X, A 450 \mathrm{~nm}$ value is dependent $\mathrm{Y}, \mathrm{R}^{2}=0.96563$. (B) $\mathrm{Y}$-values were obtained from the equation $\mathrm{Y}=-0.002536 \mathrm{X}^{2}+0.143289 \mathrm{X}+0.200338$. The mean values of 32Cys, 34Asp, 37Glu and wild-type MBL proteins were $0.980,0.971,0.900$ and $1.047 \mathrm{mg} / \mathrm{l}$, respectively. No differences were observed in the concentrations between the mutated MBL proteins and wild-type MBL $(\mathrm{P}>0.05)$.

protein (prepared in our laboratory) in TBS, anti-MBL mAb HYB131-11, and HRP-conjugated anti-mouse IgG at $37^{\circ} \mathrm{C}$ for $1 \mathrm{~h}$. The amount of proteins binding to MASP was determined by measuring A450 nm using TMB as a substrate.

C4 deposition assay. The ability of variant MBL proteins as well as wild-type MBL to activate the lectin pathway of the complement was determined by the $\mathrm{C} 4$ deposition assay. Microtitre plates were coated with mannan $(50 \mathrm{mg} / \mathrm{l})$ at $4^{\circ} \mathrm{C}$ overnight. The indicated concentrations of purified $\mathrm{MBL}$ proteins in TBST containing 5\% (w/v) skim milk were then added and incubated at $37^{\circ} \mathrm{C}$ for $1 \mathrm{~h}$. After being washed, the plates were incubated with MBL-deficiency human serum (genotype LYPB/LYPB, collected in our lab) at $37^{\circ} \mathrm{C}$ for $1 \mathrm{~h}$, anti-human C4d mAb (Quidel, San Diego, CA, USA) at $37^{\circ} \mathrm{C}$ for $1 \mathrm{~h}$, and HRP-conjugated anti-mouse $\mathrm{IgG}$ at $37^{\circ} \mathrm{C}$ for $30 \mathrm{~min}$. The amount of $\mathrm{C} 4 \mathrm{~d}$ deposition was determined by measuring A450 nm values.

Statistical analysis. Statistical significance of data was determined by comparing the results between wild-type and variant MBL proteins using a one-way analysis of variance (ANOVA) with the Tukey post hoc test. $\mathrm{P}<0.05$ was considered to denote statistical significance.

\section{Results}

Stable expression of $M B L$ genes in $\mathrm{CHO}$ cells. To investigate the biological behavior of MBL variants, we transfected pcMBLw, pcMBLm52, pcMBLm54 and pcMBLm57 into $\mathrm{CHO}$ cells by electroperforation. The same number of transfected cells was collected and identified by RT-PCR. mRNAs of variants and the wild-type MBL gene were effectively expressed in $\mathrm{CHO}$ cells (Fig. 1A). No difference was found among the MBL variants and the wild-type MBL gene (Fig. 1B).

Transient expression of MBL genes in COS-7 cells. The same mass number expression vectors of wild-type and three variants of MBL were transiently transfected into the same amount of COS-7 cells. Then, $72 \mathrm{~h}$ later, proteins secreted in the culture supernatant were analyzed by a sandwich ELISA assay. No difference of the concentrations among the variants and wild-type of MBL was found (P>0.05) (Fig. 2). 
A

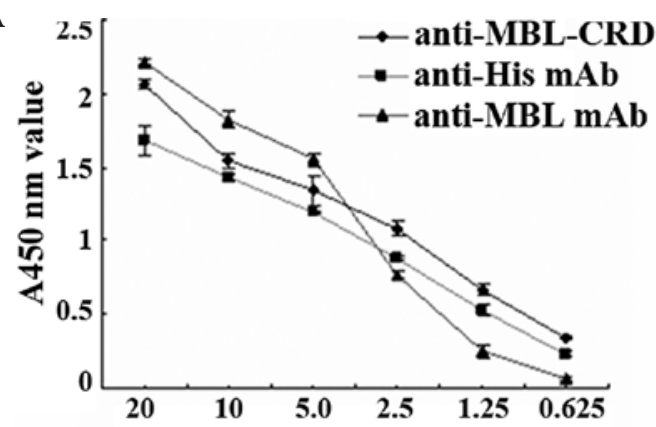

Concentration of wild-type MBL (mg/l)
B

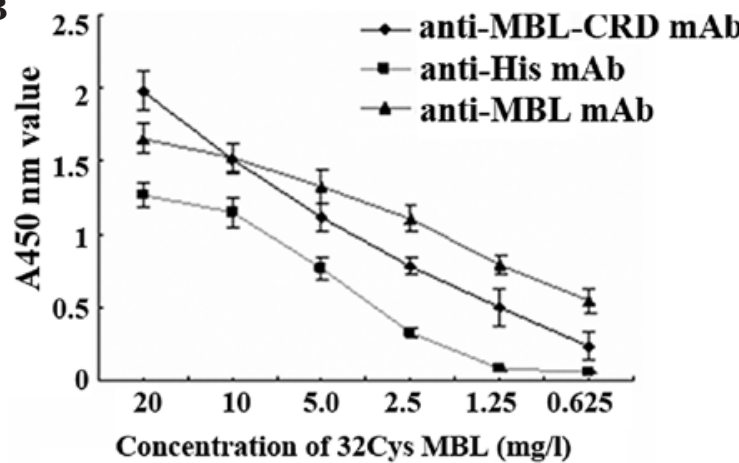

C

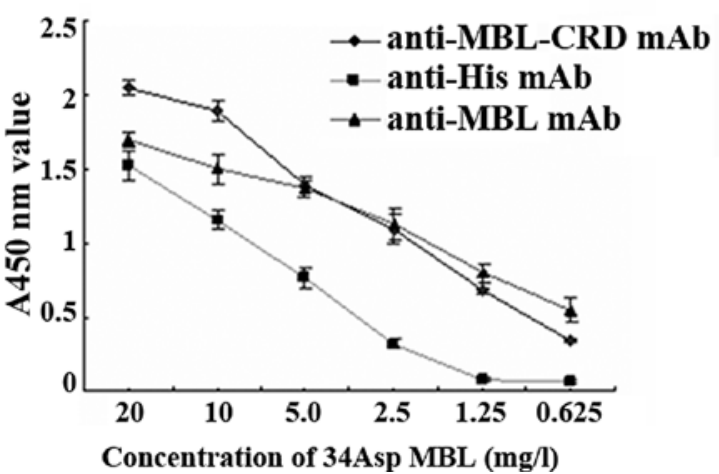

D

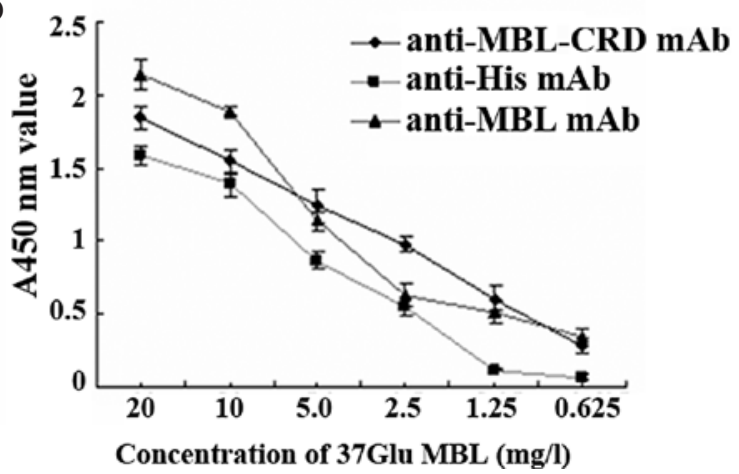

Figure 3. Identification of MBL proteins by indirect ELISA. Microtitre plates were coated with different concentrations of (A) purified recombinant wild-type MBL and (B) mutated MBL 34Asp, (C) 37Glu and (D) 32Cys. The plates were then incubated with anti-MBL-CRD, anti-His and anti-MBL mAbs, respectively, followed by HRP-conjugated anti-mouse $\mathrm{IgG}$ antibody. A450 $\mathrm{nm}$ values were measured.

A

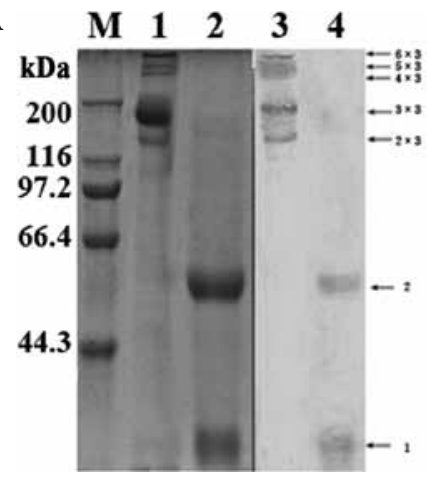

B

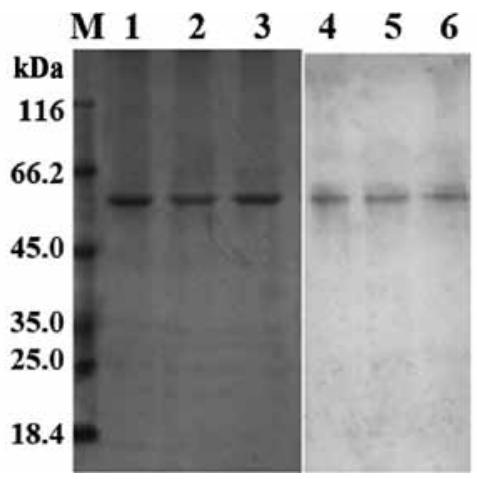

C

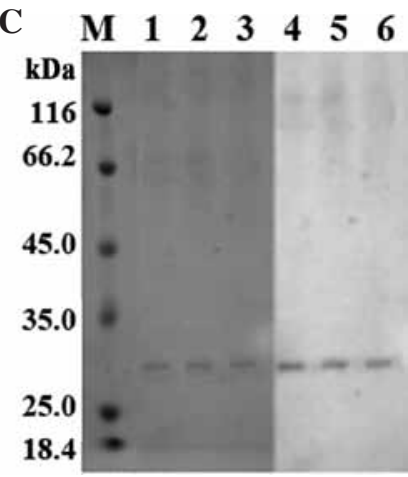

Figure 4. MBL proteins were submitted to SDS-PAGE and western blotting using anti-MBL mAb HYB 131-11 as the primary detecting antibody. (A) Recombinant wild-type MBL was analyzed under non-reducing (lanes 1 and 3) and reducing conditions (lanes 2 and 4). (B) Three variant MBL proteins were analyzed under non-reducing conditions. Lanes 1 and 4, 32Cys MBL; lanes 2 and 5, 34Asp MBL and lanes 3 and 6, 37Glu MBL. (C) Three variant MBL proteins were analyzed under reducing conditions. Lanes 1 and 4, 32Cys MBL; lanes 2 and 5, 34Asp MBL and lanes 3 and 6, 37Glu MBL.

Identification of proteins by indirect ELISA. Recombinant MBL proteins were purified and identified by indirect ELISA. Each of the proteins may be recognized by self-prepared (anti-MBL-CRD mAb) and commercialized (HYB131-11) mAbs (Fig. 3), indicating that they are MBL proteins.

Detection of MBL expression by SDS-PAGE and western blotting. The oligomeric composition of recombinant wild-type and mutated MBL proteins was analyzed on non-reduced and reduced SDS-PAGE followed by western blotting (Fig. 4). As shown in Fig. 4A, under non-reducing conditions, the recombinant wild-type MBL forms oligomers containing two, three and four chains (150, 225 and $300 \mathrm{kDa}$, respectively), whereas mutated MBL proteins form covalent oligomers containing two polypeptide chains $(\sim 60,000)$ (Fig. 4B), indicating a much lower oligomerization in the mutated MBL proteins, 32Cys, 34Asp and 37Glu. Wild-type MBL was reduced to a single chain $(25 \mathrm{kDa})$ and an oligomer containing two polypeptide chains (50 kDa) (Fig. 4A), whereas the mutated proteins were reduced to a single chain with a molecular mass of $\sim 32 \mathrm{kDa}$ (Fig. 4C). 

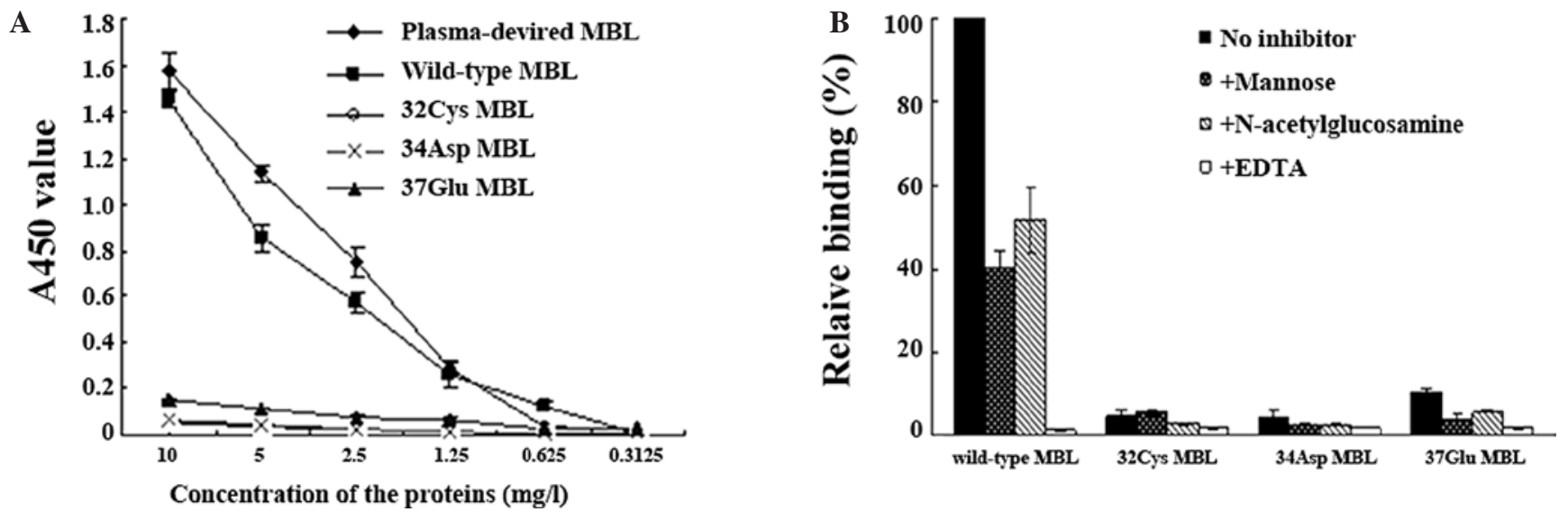

Figure 5. Analysis of binding between MBL proteins and mannan by ligand-binding ELISA. (A) Microtitre plates were coated with mannan and then incubated with plasma-derived, recombinant wild-type and mutated MBL proteins, respectively, followed by anti-MBL mAb, HRP-conjugated anti-mouse IgG antibody The amount of bound proteins was determined by measuring A450 nm values. (B) Competitive ELISA assay. Microtitre plates were coated with mannan and then incubated with recombinant wild-type and mutated MBL proteins, respectively, in the presence or absence of mannose, N-acetylglucosamine and EDTA, followed by anti-MBL mAb, HRP-conjugated anti-mouse IgG antibody. The amount of bound proteins was determined by measuring A450 $\mathrm{nm}$ values. The relative ratio was calculated by comparing the $\mathrm{A} 450 \mathrm{~nm}$ values of different groups to that of wild-type MBL without any inhibitors.

A

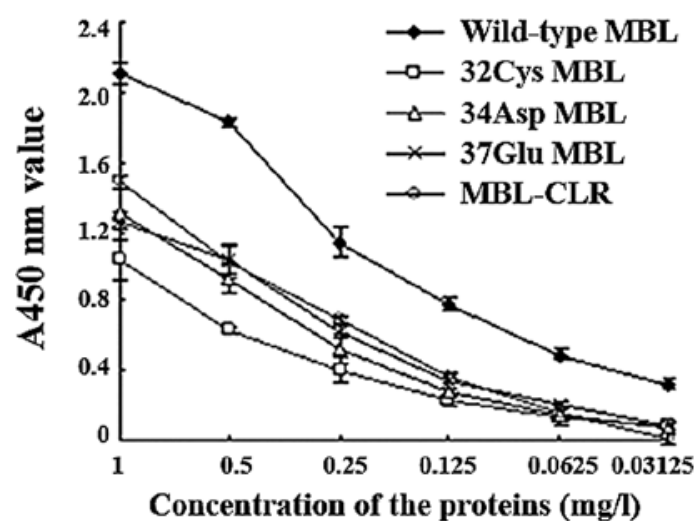

B

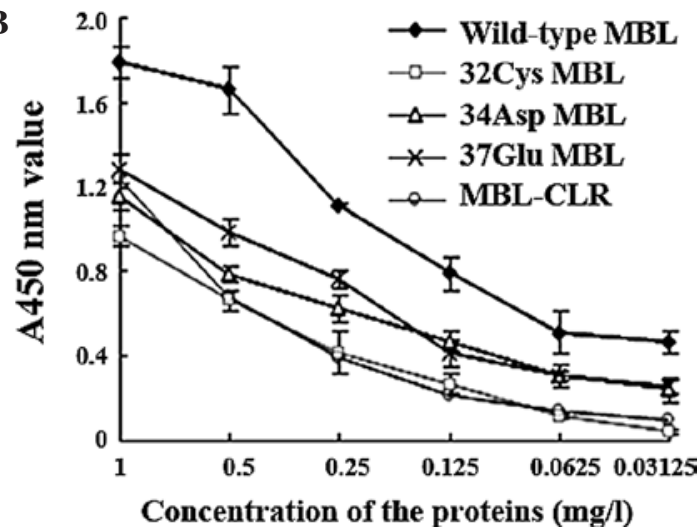

Figure 6. Analysis of binding of MBL proteins with MASPs. Microtitre plates were coated with the N-terminal fragment of (A) MASP-1 or (B) MASP-2. The plates were then incubated with recombinant wild-type MBL, mutated MBL proteins and prokaryotically expressed MBL-CLR, respectively, followed by anti-MBL mAb or anti-His mAb (for MBL-CLR protein), and HRP-conjugated anti-mouse IgG antibodies. The amount of bound proteins was determined by measuring A450 nm values.

Binding of proteins to mannan. The mannan-binding assay showed that the mutated MBL proteins lose the ability to bind to mannan (Fig. 5A), while mannose and $\mathrm{N}$-acetylglucosamine partly inhibited binding of the wild-type MBL protein to mannan, and EDTA totally inhibited the binding (Fig. 5B).

Binding of MBL to MASP as determined by ELISA. To reveal the interaction between mutated MBL and MASPs, we assessed the binding of N-terminal fragments of MASP1 and MASP2 to four MBL proteins and the MBL-CLR protein by ELISA. Three variant MBL proteins bound to MASP1 (Fig. 6A) and MASP2 (Fig. 6B) with a lower ability compared to the wild-type MBL, without, however, completely losing this binding ability.

Activities of MBL as determined by C4 cleavage assay. Not all the mutated proteins activated the complement system (Fig. 7A). Mannose and $\mathrm{N}$-acetylglucosamine partly inhibited C4d deposition resulting from the wild-type MBL, whereas EDTA completely inhibited the activity (Fig. 7B).

\section{Discussion}

The aim of the present study was to investigate the relationship among MBL gene mutations, antigenic MBL secretion levels and MBL functional activities. The MBL encoding gene was amplified by RT-PCR. The sequencing result indicated that the sequence of cDNA is identical to that of mRNA for human MBL (accession no. X15422), with the exception of the two nucleotides ACC24GCC ( $\mathrm{Thr} \rightarrow \mathrm{Ala})$ and GGC185GGT $(\mathrm{Gly} \rightarrow \mathrm{Gly})$. The nucleotide sequence was deposited in GenBank with the accession number AF360991. Using a MBL cDNA from the HepG2 human hepatocellular carcinoma cell line, Larsen et al (17) reported that mutations in the human MBL gene compromise the oligomerization and activity of the protein. We obtained MBL cDNA from the liver of a Chinese foetus, which is exists naturally. GGC54GAC, CGT52TGT and GGA57GAA variants were created with pMBLw as the template, whose sequences contain the expected point mutations without any other changes. The recombinant eukaryotic 

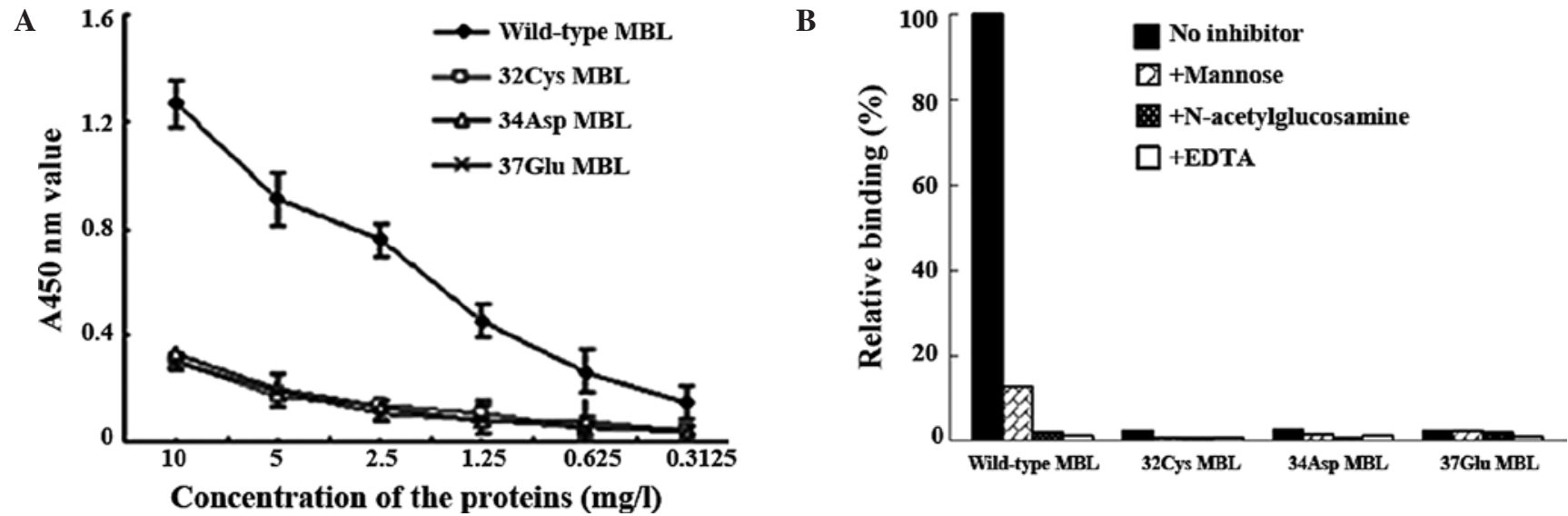

Figure 7. Effects of mutated MBL proteins on complement activation. (A) Microtitre plates were coated with mannan and then incubated with wild-type and mutated MBL proteins, respectively, followed by incubation with MBL-deficiency human serum, mouse anti-human C4d mAb. The amount of C4d deposition was determined by measuring A450 nm values. (B) Competitive C4d deposition assay. Microtitre plates were coated with mannan and then incubated with wild-type and mutated MBL proteins, respectively, in the presence or absence of mannose, N-acetylglucosamine and EDTA, followed by incubation with MBL-deficiency human serum, mouse anti-human $\mathrm{C} 4 \mathrm{~d} \mathrm{mAb}$. The amount of $\mathrm{C} 4 \mathrm{~d}$ deposition was determined by measuring A450 nm values. The relative ratio was calculated by comparing the A450 nm values of different groups to that of wild-type MBL, without any inhibitors.

expression vectors, pcMBLw, pcMBLm52, pcMBLm54 and pcMBLm57, were constructed by inserting the target sequences into eukaryotic expression vectors PcDNA4/HisMaxC, and were transfected into COS-7 and CHO cells for transient and stable expression studies, respectively.

MBL is a macroprotein with complicated functions, and it is impossible to obtain an intact protein in the protokaryon expression system. Ohtani et al (18) reported a high level and effective production of human wild-type MBL in $\mathrm{CHO}$ cells. We transfected recombinant plasmids into $\mathrm{CHO}$ cells and found that there is no difference among the mRNAs of variants. Additionally, the wild-type MBL gene is capable of being effectively expressed in $\mathrm{CHO}$ cells. From these results, we inferred that the point mutations in exon 1 of the MBL gene do not interrupt the expression of the MBL gene.

To ivestigate the effect of the mutations on the secretion of MBL protein, Larsen et al (17) attempted to achieve a transient expression in COS-7 cells; however, they failed due to low expression levels. We successfully expressed variant and wildtype MBL proteins in COS-7 cells, and gained insight into the relationship between MBL gene mutations and MBL protein synthesis or secretion, because protein levels are affected with few factors in the transient transfection system. Results of the sandwich ELISA assay showed that the gene mutations may not affect the MBL level in human plasma.

The product of the human MBL gene is a 25-32 kDa polypeptide chain containing 228 amino acids, consisting of four domains: the cysteine-rich N-terminal region responsible for the formation of intra- and inter-subunit disulfide bonds, an extended CLR (repeating Gly-X-Y triplets), a short-helical neck region initiating trimerization of the collagen-like sequence, and a C-terminal CRD that constitutes a globular head. Three identical polypeptide chains combine to form a subunit, with a tail consisting mainly of a collagen-like triple helix and a three-headed cluster of globular CRDs. Several homotrimeric subunits may then oligomerize to form a series of oligomers via disulfide bonding in the N-terminal region. The cysteinerich $\mathrm{N}$-terminal region and CLR are essential for effective oligomerization. MBL appears to exist in plasma as a mixture of 2-6 trimeric subunits. Only high-order oligomers (teramers or larger oligomers) interact with carbohydrates with higher affinity and efficiently activate the complement. The common character of the three mutations is that all of them affect the Gly-X-Y repeats in the CLR (17).

To further study the relationship between gene mutations and the protein activities of human MBL, we purified recombinant wild-type as well as mutated MBL proteins and found that mutated MBL has less oligomerization compared to wildtype MBL. Mutated MBL proteins lose the mannan binding and complement activation ability. Binding of MBL to MASPs showed that the three variant MBL proteins bind to MASP1 and MASP2 with a lower ability compared to wild-type MBL, although they do not completely lose this binding ability. This gives rise to the possibility that the three point mutation sites are not involved in the binding of MASPs. This assumption is consistent with the finding that the mutation sites of MBL are not involved in the binding sites for MASPs, as demonstrated by our group using synthetic peptides (19). Therefore, the inability of the variant MBL proteins to activate the complement results from lower oligomerization, which impairs the interaction of MBL with mannan and MASPs. As for CGT52TGT, a Cys introduced by the mutation may form another disulfide bond that may disrupt the structure of the MBL molecule, as well as its function. GGC54GAC and GGA57GAA mutations introduce Asp or Glu with a large side chain and charge instead of neutral Gly, and may affect formation of the CLR $\alpha$-helix, resulting in the formation of fewer oligomers.

Since Valdimarsson et al (20) reported that MBL replacement therapy with a plasma-derived product is both safe and promising, attention has focused on MBL in clinical research. Terms such as 'MBL deficiency' and 'MBL insufficiency' have not been well-defined due to high variability in different individuals. The plasma levels of MBL are affected by various factors, such as age, race, gene polymorphism and individual status. In individuals with the same MBL genotype, a MBL concentration of five times or more exists. Therefore, susceptibility to infections may not be affected simply by the plasma MBL levels. Furthermore, it was reported that variant 
alleles in humans give rise to relatively high levels of $\mathrm{MBL}$ in the circulation (21). MBL deficiency does not only involve lower MBL levels, but also structural changes. In this study, we found that the variant forms of MBL differ in the forms of oligomers. These variant forms all form a dominant band with a molecular mass of approximately $60 \mathrm{kDa}$, responding in the decrease of their functions. However, we did not find that these point mutations affect the synthesis or secretion of the MBL protein. Immunodeficiency caused by MBL mutations may be the result of less oligomerization, rather than the decrease in plasma MBL levels. Therefore, we noted that the immunological methods based on the antigen-antibody binding may not distinguish normal MBL from variant MBL. The most important tool for the dissection of MBL deficiency involves two ELISA systems that detect MBL levels; one is based on the double-antibody sandwich ELISA, whereas the other is the mannan-binding ELISA. The former detects all the forms of MBL, including monomers and oligomers, while the latter only detects MBL oligomers, but may be interfered with by classical pathway activation by anti-mannan antibodies (22). Special consideration with these ELISA systems should be given to the detection of polymers as opposed to various forms of MBL, in order that the actual levels of functional MBL are reflected.

In conclusion, three forms of MBL gene mutations decrease the functions of their products, including the binding to mannan and MASPs and the ability to activate the complement, which result from lowered protein oligomerization, although they do not affect the synthesis or secretion of the MBL protein. The data suggest that MBL deficiency associated with three polymorphic variants may result from impaired oligomerization of the MBL protein rather than the decrease of MBL levels in plasma. Additionally, amino acids Arg32, Gly34 and Gly37 are required to maintain the structure and function of MBL.

\section{Acknowledgements}

This study was supported by the Natural Scientific Foundation of China (39970286), and the Natural Scientific Foundation Team Research Project of Guangdong Province (015003).

\section{References}

1. Gupta G and Surolia A: Collectins: sentinels of innate immunity. Bioessays 29: 452-464, 2007.

2. Turner MW: The role of mannose-binding lectin in health and disease. Mol Immunol 40: 423-429, 2003.

3. Dommett RM, Klein N and Turner MW: Mannose-binding lectin in innate immunity: past, present and future. Tissue Antigen 68: 193-209, 2006

4. Jack DL, Klein NJ and Turner MW: Mannose-binding lectin: targeting the microbial world for complement attack and opsonophagocytosis. Immunol Rev 180: 86-99, 2001.
5. Hajela K, Kojima M, Ambrus G, et al: The biological functions of MBL-associated serine proteases (MASPs). Immunobiol 205: 467-475, 2002.

6. Bohlson SS, Fraser DA and Tenner AJ: Complement proteins $\mathrm{Clq}$ and MBL are pattern recognition molecules that signal immediate and long-term protective immune functions. Mol Immunol 44: 33-43, 2007.

7. Eisen DP and Minchinton RM: Impact of mannose-binding lectin on susceptibility to infectious diseases. Clin Infect Dis 37: 1496-1505, 2003.

8. Worthley DL, Bardy PG and Mullighan CG: Mannose-binding lectin: biology and clinical implications. Intern Med J 35: 548-555, 2005.

9. Monticielo OA, Mucenic T, Xavier RM, Brenol JC and Chies JA: The role of mannose-binding lectin in systemic lupus erythematosus. Clin Rheumatol 27: 413-419, 2008.

10. Maury CP, Aittoniemi J, Tiitinen S, Laiho K, Kaarela K and Hurme M: Variant mannose-binding lectin 2 genotype is a risk factor for reactive systemic amyloidosis in rheumatoid arthritis J Intern Med 262: 466-469, 2007.

11. Carlsson M, Sjöholm AG, Eriksson L, Thiel S, Jensenius JC, Segelmark $\mathrm{M}$ and Truedsson L: Deficiency of the mannanbinding lectin pathway of complement and poor outcome in cystic fibrosis: bacterial colonization may be decisive for a relationship. Clin Exp Immunol 139: 306-313, 2005.

12. Foster CB, Lehrnbecher T, Mol F, et al: Host defense molecule polymorphisms influence the risk for immune-mediated complications in chronic granulomatous disease. J Clin Invest 102: 2146-2155, 1998

13. Garred P, Larsen F, Seyfarth J, Fujita R and Madsen HO: Mannose-binding lectin and its genetic variants. Genes Immun 7: 85-94, 2006.

14. Yu XP, Lv CW, Ge ZM, Li JC, Ma L and Chen ZL: Investigation of single nucleotide polymorphisms, haplotypes and genotypes of mannan-binding lectin gene in Bai (Pai) nationality in China. Chinese J Immunol (in Chinese) 22: 738-742, 2006.

15. Zhong CQ, Yu XP, Wang FY, Kurexijiang T and Chen ZL: Frequencies of CGT52TGT, GGC54GAC and GGA57GAA point mutations in MBL gene in Chinese Uyghur population. Nan Fang Yi Ke Da Xue Xue Bao (In Chinese) 26: 1764-1767, 2006.

16. Petersen SV, Thiel S and Jensenius JC: The mannan-binding lectin pathway of complement activation: biology and disease association. Mol Immunol 38: 133-149, 2001.

17. Larsen F, Madsen HO, Sim RB, Koch C and Garred P: Disease-associated mutations in human mannose-binding lectin compromise oligomerization and activity of the final protein. J Biol Chem 279: 21302-21311, 2004.

18. Ohtani K, Suzuki Y, Eda S, et al: High-level effective production of human mannan-binding lectin MBL in Chinese hamster ovary CHO cells. J Immunol Methods 222: 135-144, 1999.

19. Zuo DM, Cai XM, Zhao N, Zhang LY and Chen ZL: Location of MBL-associated serine proteases binding motifs on human mannan-binding lectin (MBL). Protein Peptide Lett 17: 131-136, 2010.

20. Valdimarsson H, Stefansson M, Vikingsdottir T, Arason GJ, Koch C, Thiel S and Jensenius JC: Reconstitution of opsonizing activity by infusion of mannan-binding lectin (MBL) to MBL-deficient humans. Scand J Immunol 48: 116-123, 1998.

21. Garred P, Larsen F, Madsena HO and Koch C: Mannose-binding lectin deficiency - revisited. Mol Immunol 40: 73-84, 2003.

22. Seelen MA, Roosa A, Wieslander J, et al: Functional analysis of the classical, alternative, and MBL pathways of the complement system: standardization and validation of a simple ELISA. J Immunol Methods 296: 187-198, 2005. 